\title{
Study on low-frequency dielectric behavior of the carbon black/polymer nanocomposite
}

H. Shivashankar ${ }^{1, *}$ (I), Kevin Amith Mathias ${ }^{2}$, Pavankumar R. Sondar ${ }^{3}$, M. H. Shrishail ${ }^{4}$, and S. M. Kulkarni ${ }^{5}$

\footnotetext{
${ }^{1}$ Department of Mechatronics, Manipal Institute of Technology, Manipal Academy of Higher Education, Manipal, Karnataka 576104, India

${ }^{2}$ Department of Mechanical and Manufacturing Engineering, Manipal Institute of Technology, Manipal Academy of Higher Education, Manipal, Karnataka 576104, India

${ }^{3}$ Department of Metallurgical and Materials Engineering, National Institute of Technology, Karnataka Surathkal, Mangalore 575025, India

${ }^{4}$ Department of Electronics and Communication Engineering, National Institute of Technology, Rourkela, Odisha 769008, India

${ }^{5}$ Department of Mechanical Engineering, National Institute of Technology, Karnataka Surathkal, Mangalore 575025, India
}

Received: 27 March 2021

Accepted: 12 October 2021 Published online:

31 October 2021

(C) The Author(s) 2021

\begin{abstract}
Recently, polymer-based dielectric materials have become one of the key materials to play an essential role in clean energy production, energy transformation, and energy storage applications. The end usage is the energy storage capability because it is a trade-off between dielectric permittivity, dielectric loss, and dissipation factor. Hence, it is of prime importance to study the dielectric properties of polymer materials by adding filler material at a low-frequency range. In the present study, polydimethylsiloxane/carbon black nanocomposites are prepared using the solution cast method. The dielectric properties, such as dielectric constant, dielectric loss, and dissipation factors due to the concentration of filler particles and low-frequency effect on the nanocomposites, are examined. Also, different empirical models are used to estimate the dielectric permittivity of polymer nanocomposites. The low-frequency range of $100 \mathrm{~Hz}$ to $1 \mathrm{MHz}$ and the effect of varying volume fractions of carbon black show a significant change in the dielectric properties. It is found that the nanocomposites have a higher dielectric permittivity than the base polymer material. It is also observed that an increase in filler concentration increases the dielectric permittivity, which is confirmed with an empirical model.
\end{abstract}




\section{Introduction}

In the present research world, electromagnetic interactions with solid and liquid dielectric materials are of potential interest. In the current scenario, a huge number of devices operate through the interaction of the electromagnetic wave with dielectric materials. However, characterization of the interface and interaction between fields and material is a critical task in an electromagnetic device. Although significant efforts are made in studying the interaction of waves with the material [1-3], there is a scope for electromagnetic field investigation on dielectric material as to how a time-varying electric field interacts with materials. The electromagnetic absorbance performance of the materials provides good electromagnetic properties, such as impedance matching and dielectric loss. The dielectric loss is the permittivity parameter that plays a vital role in attenuating electromagnetic energy [4]. Hence, the study of the dielectric behaviors of the material becomes necessary. The electromagnetic absorption capacities of the materials are related to the complex permittivity. The complex permittivity depends on the dielectric properties, such as dielectric constant and dielectric loss. To determine dielectric properties, energy storage and dissipation capability are the essential parameters in advanced materials [5-7]. Also, the loss tangent is another critical property used to estimate the dielectric loss of the absorbing material [8].

In the recent past, many researchers have focused on the fabrication and characterization of composite materials with enhanced electromagnetic responses. Considering metal, ceramic, and polymer materials, polymer materials are gaining popularity because of their large electric breakdown field, low dielectric loss, easy fabrication, and cost-effectiveness [9, 10]. However, the dielectric constant of the common polymer materials is very low. Thus, enhancing the polymer's dielectric properties by retaining other excellent properties is a challenging task [11, 12]. One of the approaches to increasing the dielectric properties is introducing a filler material with an electrically conductive particle. The polymer filled with conductive material is essential for energy storage applications. Many studies have reported the significance of conducting filler with respect to the dielectric properties of polymer nanocomposite
$[13,14]$. The dielectric properties of polymer nanocomposites depend on the type of material used, size, and shape of the filler used in the main matrix material. In general, electrically conductive nanocomposite materials are used in the sensing electrode, sensor and actuator, pressure/temperature sensor, and electronic applications [15-17]. Another important way to estimate the dielectric properties of the nanocomposite is the empirical approach. Few researchers have shown the dielectric behavior of polymer nanocomposites using experimental data and mixing rules [18, 19]. Araújo et al. have used the different theoretical models to evaluate the dielectric behavior of the ceramic and polymer composite. They have used PZT/poly(vinylidene fluoride) and $\mathrm{BaTiO} /$ poly(vinylidene fluoride) composites with varying filler concentrations. The main interaction of composites dielectric behavior is studied and estimated with a suitable model [20]. Zhang et al. have used the polydimethylsiloxane (PDMS) polymer filled with zinc oxide $(\mathrm{ZnO})$ powders to study the dielectric and piezoelectric properties. The dielectric constant of these materials is estimated through different theoretical approaches [21]. Nawanil et al. have explored the electrical properties of barium titanate/ PDMS nanocomposites. They have also fabricated and studied the BT/PDMS nanocomposites modified with carbon nanotube (CNT). Different theoretical models were used to predict the dielectric constant of these composites and compare their experimental value with the theoretical value [22].

In the present study, preparation of nanocomposite material, characterization of the dielectric properties at low-frequency range, and different empirical models are discussed. PDMS is one of the matrix materials used primarily for dielectric applications $[23,24]$. The conductive filler, such as carbon black particles, has been used to enhance the nanocomposite's dielectric properties. The nanocomposite sample is synthesized with a varied volume percentage of carbon black. The plain polymer (PDMS) and PDMS/CB nanocomposites' dielectric properties are measured using an LCR meter at a low-frequency range. The dielectric permittivity of the nanocomposites data is collected experimentally and compared with the well-defined empirical models. Therefore, the present approach investigates the dielectric properties of pure PDMS and PDMS/CB 
nanocomposite material at a low-frequency range, which can be used for electronic applications.

\section{Dielectric analysis}

The dielectric material can store the electrical charge due to the applied electric field. The polarization and electron distributions occur through the material by supplying the alternating current. The capacitance and tangent loss are measured by changing the sweep frequency $[25,26]$. Hence, the dielectric permittivity $\left(\varepsilon^{\prime}\right)$ of the nanocomposite can be calculated using Eq. 1.

$\varepsilon^{\prime}=\frac{C_{m} t}{\varepsilon_{0} A}$

The measured capacitance is $C_{m}, t$ is the thickness of the sample, $\varepsilon_{0}$ is the dielectric permittivity of the vacuum $(8.854 \mathrm{pF} / \mathrm{m})$, and $A$ is the effective area. The dielectric loss results from the inability of a polarization process in the material due to the applied electric field. Generally, the loss in a material is expressed in terms of the loss tangent. Thus, the loss tangent is used to calculate the dielectric loss $\left(\varepsilon^{\prime}\right)$ of the nanocomposite materials using Eq. 2.

$\tan \delta=\frac{\varepsilon^{\prime \prime}}{\varepsilon^{\prime}}$

The loss tangent $(\tan \delta)$ can also be represented as dissipation factor and loss factor. The complex permittivity of the material can be expressed as given in Eq. 3.

$\varepsilon^{*}=\varepsilon^{\prime}-i \varepsilon^{\prime \prime}$

The magnitude of the real component is also referred to as the dielectric constant $\left(\varepsilon^{\prime}\right)$ and the magnitude of the imaginary part is also called dielectric loss $\left(\varepsilon^{\prime \prime}\right)$. The polarization of the material is dependent on the varied frequency under an applied electric field.

\section{Empirical models}

Some empirical approaches were used to estimate the effective dielectric constant of the polymer nanocomposites. The models were consolidated in the present study based on an approximation of the mean field of effective permittivity of the nanocomposite materials [27-31].

\subsection{Maxwell-Garnett model}

Maxwell-Garnett's rule addresses the mix rule to determine the effect of the second phase of the composite's dielectric properties. The model is predicted based on the homogeneous and isotropic dielectric permittivity of the matrix material $\left(\varepsilon_{m}\right)$ and dielectric permittivity of the filler material $\left(\varepsilon_{f}\right)$. The volume fraction of the filler particle is $v_{f}$ and the resulting volume fraction of the matrix is $\left(v_{m}=1-v_{f}\right)$. It is assumed that the nanocomposite component has no dielectric loss in the interested region of frequency. The Maxwell-Garnett mixing rule is perhaps the most widely applied in part because of its simplicity for the two-phase mixture, as written in Eq. 4.

$\varepsilon_{c}=\varepsilon_{m}\left[1+\frac{3 v_{f}\left(\varepsilon_{f}-\varepsilon_{m}\right)}{\left(1-v_{f}\right)\left(\varepsilon_{f}-\varepsilon_{m}\right)+3 \varepsilon_{m}}\right]$

The effective dielectric permittivity $\left(\varepsilon_{c}\right)$ of the nanocomposite is essentially summing the individual polarizability of distinct particle inclusions. Generally, the particle's spherical (or ellipsoidal) shape helps to accurately calculate the induced field within the background host material, which is then approximately modified to account for the interactions between the inclusions.

\subsection{Bruggeman model}

Bruggeman proposed an improved version of the mixing rule for a low volume fraction of the filler to be gradually increased for a higher volume addition. It is particularly suitable for a disordered system. The constituent particles may become very close to each other and even agglomerate so that the deviations from the ideally uniform and dilute system may be substantial even at a low filler concentration. For the denser composite, Bruggeman's theory works better than the other rules. The relative dielectric approximate of the Bruggeman equation is expressed in Eq. 5

$\varepsilon_{c}=\varepsilon_{f}\left[\frac{3 \varepsilon_{m}+2 v_{f}\left(\varepsilon_{f}-\varepsilon_{m}\right)}{3 \varepsilon_{f}-v_{f}\left(\varepsilon_{f}-\varepsilon_{m}\right)}\right]$

\subsection{Lichtenecker's logarithmic model}

Lichtenecker's logarithmic rule is one of the wellknown models for predicting the dielectric permittivity of the nanocomposite. It can only be used when 
the spatial distribution of the filler particles' shape and orientation is random. The effective dielectric permittivity of the nanocomposite is dependent on how well it satisfies Lichtenecker logarithmic model. The detailed study on Lichtenecker logarithmic mixing rule is expressed in Simpkin R's report [32] and the modified logarithmic law is written in Eq. 6

$\varepsilon_{c}=\varepsilon_{m}^{1-v_{f}} \cdot \varepsilon_{f}^{v_{f}} \cdot\left(\frac{\varepsilon_{m}}{\varepsilon_{f}}\right)^{u v_{f}}$

The parameter ' $u$ ' is introduced for the mixing law to optimize the matrix and filler material ratio. The value ' $u$,' frequently used in the literature, varies from 0 to 3 for the nanocomposites with the shape of the filler particles. The experimentally determined value is 0.3 for well-dispersed nanocomposite material.

\subsection{Wiener model}

The two phases of the nanocomposite effective dielectric permittivity can be evaluated by Eq. 7 . This model can provide the upper and lower bound for the dielectric constant. The model mainly depends on the uniform distribution of the particle and continuous conditions at the material interface [33, 34].

$\frac{1}{\varepsilon_{c}}=\frac{v_{f}}{\varepsilon_{f}}-\frac{1-v_{f}}{\varepsilon_{m}}$

\subsection{Knott model}

Knott introduced a model in which the filler particle is considered as a small cube surrounded by matrix material with equal dimensions [35]. The effective dielectric constant can be determined using Eq. 8.

$\varepsilon_{c}=\varepsilon_{m}\left[1-\frac{\left(\varepsilon_{m}-\varepsilon_{f}\right) v_{f}}{\varepsilon_{f}+\left(\varepsilon_{m}-\varepsilon_{f}\right) v_{f} 0.33}\right]$

\subsection{HS-CT model}

Hashin and Shtrikman's (HS) model is used to calculate the effective permeability of the multiphase magnetic material [36]. The HS method is modified and used by Cule and Torquato (CT), and it has adopted the same approach that was developed for the dielectric permittivity of the composite material [37]. The assumptions were made considering the cylindrical shape matrix with filler material at its core. The model mainly depends on the radius of the matrix and filler material. The useful dielectric constant HS-CT model is represented in Eq. 9.

$\varepsilon_{c}=\varepsilon_{m} \frac{\varepsilon_{f}+\varepsilon_{m}+\left(\varepsilon_{f}-\varepsilon_{m}\right) v_{f}}{\varepsilon_{f}+\varepsilon_{m}-\left(\varepsilon_{f}-\varepsilon_{m}\right) v_{f}}$

\subsection{Yamada model}

Yamada et al. have developed a model for the dielectric and piezoelectric properties of the composites [38]. The two phases of the composites are uniformly dispersed with ellipsoidal particles in the continuous medium. The model is derived using the static field conditions. The parameter ' $n$ ' is introduced in the model as shown in Eq. 10. It depends on the shape orientation of the ellipsoidal filler particles. The constant value is more prominent than one and it depends on the experimental conditions.

$\varepsilon_{c}=\varepsilon_{m}\left[1+\frac{n\left(\varepsilon_{f}-\varepsilon_{m}\right) v_{f}}{n \varepsilon_{m}+\left(1-v_{f}\right)\left(\varepsilon_{f}-\varepsilon_{m}\right)}\right]$

\subsection{Nielsen model}

The Nielsen model mainly depends on the way filler particles are packed in the composite material [39]. Thus, the model introduces a new parameter, ' $k$,' to obtain the effective dielectric constant of the composite. Parameter ' $k$ ' depends on the maximum packaging factor and the shape of the filler particle, i.e., $k=0.63$ for random packaging and $k=0.52$ for cubic packaging of the spheres. The term ' $s$ ' shown in Eq. 11 is the particle's polarization factor that depends on the shape of the filler particle, i.e., $s=3$ for the sphere.

$$
\begin{gathered}
\varepsilon_{c}=\varepsilon_{m}\left[\frac{1+s B v_{f}}{1-A B v_{f}}\right] \\
A=1+\frac{1-k}{k^{2}} v_{f} \text { and } B=\frac{\left[\frac{\varepsilon_{f}}{\varepsilon_{m}}-1\right]}{\left[\frac{\varepsilon_{f}}{\varepsilon_{m}}+s\right]}
\end{gathered}
$$

\subsection{Jayasundere and Smith model}

Jayasundere-Smith's model comprises the interaction between neighboring spheres. This model involves the electric field of the dielectric particle embedded in the continuous dielectric medium, i.e., matrix 
material. The polarization takes place in the adjacent particle [40]. Thus, the expression used for effective dielectric calculation uses the average electric fields in each constituent as represented in Eq. 12.

$$
\begin{gathered}
\varepsilon_{c}=\frac{\varepsilon_{m}\left(1-v_{f}\right)+\varepsilon_{f} v_{f} M N}{\left(1-v_{f}\right)+v_{f} M N} \\
M=\frac{3 \varepsilon_{m}}{\left(2 \varepsilon_{m}+\varepsilon_{f}\right)} \quad \text { and } \quad N=1+3 v_{f} \frac{\left(\varepsilon_{f}-\varepsilon_{m}\right)}{\left(2 \varepsilon_{m}+\varepsilon_{f}\right)}
\end{gathered}
$$

\subsection{Poon and Shin model}

Poon and Shin's model is a simple explicit equation for estimating the effective dielectric constant of the composite material [41]. The model depends on the insertions that need to be uniformly distributed and separated from each other. This model overcomes some inconsistencies in the Jayasundere and Smith models. The derived model of the composite for the two conditions of $\varepsilon_{m} \geq \varepsilon_{f} a n d \varepsilon_{f} \geq \varepsilon_{m}$ is shown in Eq. 13 .

$\varepsilon_{c}=\varepsilon_{m}\left[1+\frac{\left(\frac{\varepsilon_{f}}{\varepsilon_{m}}-1\right) v_{f}}{v_{f}+0.33\left(1-v_{f}\right)\left[\frac{\varepsilon_{f}}{\varepsilon_{m}}\left(1-v_{f}\right)+v_{f}+2\right]}\right]$

In order to examine the influence of conductive filler on the composite material, various empirical models were used to predict the effective dielectric constant. Among these models, few of the models were successful in predicting the dielectric behavior of the nanocomposite.

\section{Material and methods}

\subsection{Materials}

The material used in the present study is silicone elastomer, purchased from Dow Corning Ltd. The elastomer material has a two-part liquid component, consisting of a monomer base material and a crosslinker. They are mixed at a weight ratio of 10:1 to make a silicon-based polymer. The carbon black filler material of Vulcan XC72 is received from Cabot Corporation and is used for experiments without further processing.

The procured materials are used for the synthesis of the nanocomposite sample. Some of the properties of PDMS and carbon black are mentioned in Table 1.

\subsection{Sample preparation}

The polymer samples are prepared by adding the monomer compound into the cross-linker as recommended by the manufacturer. Then 8 to $10 \mathrm{~min}$ of shear mixing using a motor-based stirrer can fuse the cross-linker in the monomer evenly. It is then followed by degassing for 20 to $30 \mathrm{~min}$ and held in a vacuum chamber to eliminate trapped bubbles in the composite. The degasified mixture is then poured into the metal-based mold. The samples are cured at room temperature for $48 \mathrm{~h}$. The healed PDMS is then taken off from the mold and subjected to dielectric study.

The same step is to be followed by adding a varied volume fraction of carbon black particles into the pure PDMS to prepare polymer nanocomposite. All the fabrication steps are detailed in Fig. 1.

\subsection{Measurement technique}

The impedance technique can be used to analyze the dielectric properties of polymer nanocomposites. The impedance of pure PDMS and nanocomposites with varying $5 \%, 15 \%$, and $25 \%$ carbon black is measured using a precision LCR meter, E4980 (Agilent Technologies). A lab-made setup is built as per the ASTM D150 standard to determine the nanocomposite material's capacitance and loss tangent. A scanning electron microscope observes the nanocomposites' surface morphology (SEM), model-Jeol JSM-6380L.

The nanocomposite sample is fabricated in a cylindrical shape with a diameter of $12 \mathrm{~mm}$ and a thickness of $3 \mathrm{~mm}$. The developed PDMS/CB nanocomposite with a varying volume percentage of carbon black is shown in Fig. 2. Circular surface of both sides is polished and coated with a thin layer of silver to act as an electrode. The measurement is carried out in a low-frequency range of $100 \mathrm{~Hz}$ to $1000 \mathrm{kHz}$. Samples of pure PDMS and PDMS/CB nanocomposites are tested in a parallel plate mode using the LCR meter.

\section{Results and discussion}

The effective dielectric constant, dielectric loss, and dissipation factor for pure PDMS and PDMS/CB nanocomposites are discussed in the present section. The interpretation of the dielectric constant is 
Table 1 Properties of PDMS and CB material [42]

\begin{tabular}{lllllll}
\hline Material & $\begin{array}{l}\text { Young modulus } \\
(E) \mathrm{MPa}\end{array}$ & $\begin{array}{l}\text { Density }(\rho) \\
\mathrm{kg} / \mathrm{m}^{3}\end{array}$ & $\begin{array}{l}\text { Poison's } \\
\text { ratio }(v)\end{array}$ & $\begin{array}{l}\text { Thermal conductivity } \\
(\mathrm{k}) \mathrm{W} /(\mathrm{m} \mathrm{K})\end{array}$ & $\begin{array}{l}\text { Coefficient of thermal } \\
\text { expansion }(\alpha)(1 / \mathrm{K})\end{array}$ & $\begin{array}{l}\text { Dielectric } \\
\text { permittivity }(\varepsilon)\end{array}$ \\
\hline PDMS & 1.84 & 1030 & 0.45 & 0.27 & $310 \times 10^{-6}$ & $2.4-2.6$ \\
$\mathrm{CB}$ & 10.04 & 1890 & 0.27 & NA & $9 \times 10^{-6}$ & NA \\
\hline
\end{tabular}

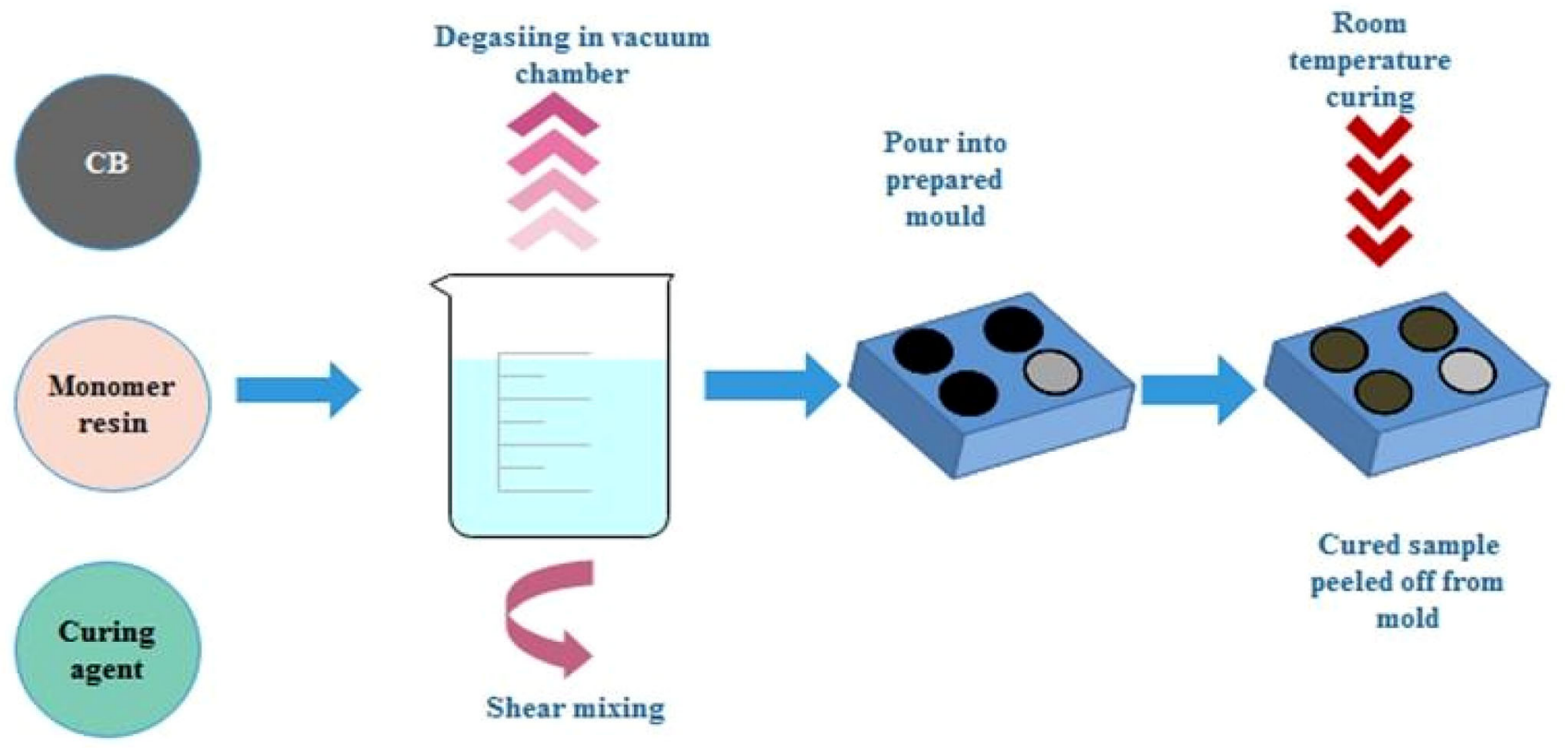

Fig. 1 Preparation steps of PDMS/CB nanocomposite material

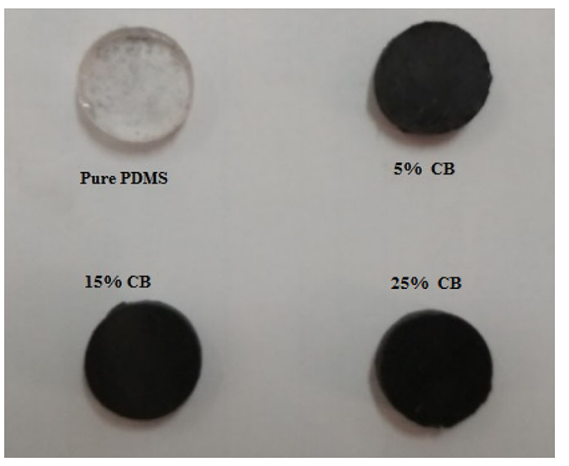

Fig. 2 Composites samples of plain PDMS and different volume percentages of $\mathrm{CB}$

justified by the use of morphology and prediction model studies.

\subsection{Dielectric constant $\left(\varepsilon^{\prime}\right)$}

The term permittivity is used to describe a material's ability to store a charge when used as a dielectric material. This represents the effect of the conductive particle and frequency dependence on the dielectric constant. The measured dielectric constant of the low-frequency range of pure PDMS and PDMS/CB nanocomposites data is given in Table 2.

\subsubsection{Effect of volume fraction}

The dielectric constant is an essential property in the development of electronic applications. Variation of dielectric constant with the increase in the carbon black volume fraction is shown in Fig. 3. As CB content increases from 5 to $25 \%$, there is a steady rise in the dielectric constant. There is a slight rise at a lower frequency, while at a medium frequency, there is a steady increase and a sudden increase in dielectric constant at a higher frequency. It indicates an increased dielectric constant in the medium, and a higher frequency could be due to the higher concentration of the conductive filler. 
Table 2 Dielectric properties of pure PDMS and PDMS/CB nanocomposites

\begin{tabular}{llll}
\hline Material & Frequency & Dielectric constant & Dielectric loss \\
\hline Pure & $100 \mathrm{~Hz}$ & 2.023 & -0.03512 \\
PDMS & $1 \mathrm{kHz}$ & 2.605 & 0.01697 \\
& $10 \mathrm{kHz}$ & 2.585 & 0.00651 \\
& $100 \mathrm{kHz}$ & 2.586 & 0.00302 \\
$5 \% \mathrm{CB}+$ PDMS & $1 \mathrm{MHz}$ & 2.651 & 0.02478 \\
& $100 \mathrm{~Hz}$ & 2.200 & -0.08756 \\
& $1 \mathrm{kHz}$ & 2.636 & 0.02673 \\
& $10 \mathrm{kHz}$ & 2.635 & 0.00793 \\
$15 \% \mathrm{CB}+$ PDMS & $100 \mathrm{kHz}$ & 2.633 & 0.00456 \\
& $1 \mathrm{MHz}$ & 2.695 & 0.03019 \\
& $100 \mathrm{~Hz}$ & 2.443 & -0.08007 \\
& $1 \mathrm{kHz}$ & 2.832 & 0.03023 \\
$25 \% \mathrm{CB}+$ PDMS & $10 \mathrm{kHz}$ & 2.812 & 0.0086 \\
& $100 \mathrm{kHz}$ & 2.810 & 0.00541 \\
& $1 \mathrm{MHz}$ & 2.881 & 0.03232 \\
& $100 \mathrm{~Hz}$ & 2.910 & 0.02931 \\
& $1 \mathrm{kHz}$ & 3.623 & 0.03952 \\
& $10 \mathrm{kHz}$ & 3.586 & 0.01243 \\
& $100 \mathrm{kHz}$ & 3.580 & 0.06909 \\
\hline
\end{tabular}

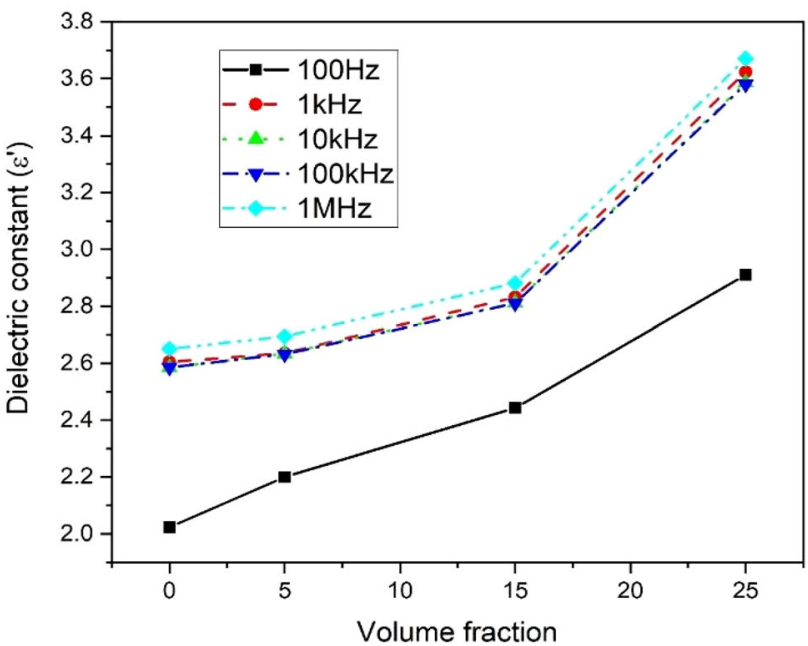

Fig. 3 Variation of dielectric constant as a function of the change in volume fraction

The nanocomposite material is electrically heterogeneous. There is also an interfacial polarization that can occur. Interfacial polarization leads to an increase in the dielectric constant due to the movement of charges. In the present analysis, two different phases of materials are used to make a nanocomposite structure. The charge link is created in the nanocomposite by adding the conductive material into the plane polymer material, which may help to trap the charges. The carbon black increases the polarization and forms the nanocomposite's bonding, increasing the dielectric constant. The increase in the nanocomposite's dielectric constant can be attributed to the increased filler volume fraction. A considerable rise in dielectric constant is noted for $25 \%$ CB content in the present investigation.

\subsubsection{Effect of frequency}

The dielectric constant of pure PDMS and PDMS/CB composite vs. frequency is shown in Fig. 4 . It is found

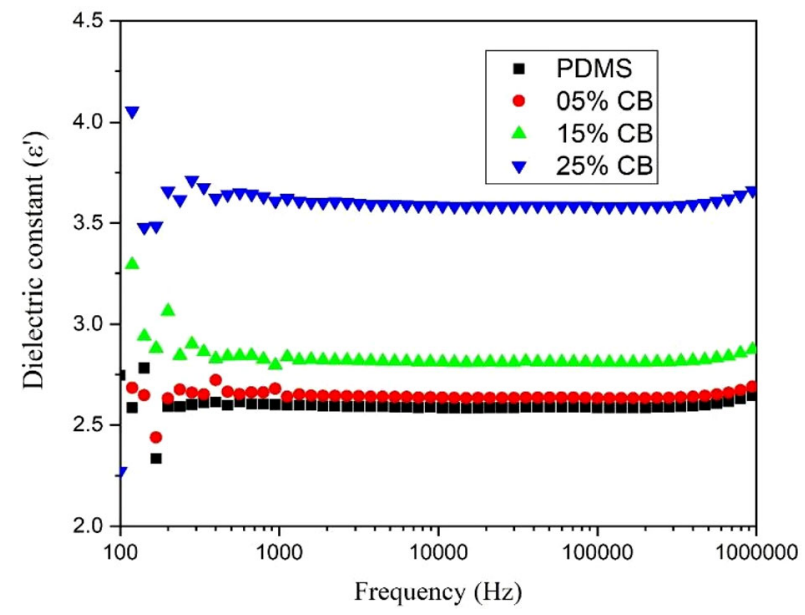

Fig. 4 Variation of dielectric constant as a function of the frequency 
that the polymer composite with lower $\mathrm{CB}$ content $(5 \%)$ is not sensitive to $A C$ frequency. However, with the increase in CB filler (15\%), the content polymer becomes slightly sensitive to low frequency. Further, with the higher CB content (25\%), the dielectric constant increases significantly with the increased frequency.

\subsection{Dielectric loss $\left(\varepsilon^{\prime \prime}\right)$}

Once the electric field is applied, it becomes difficult to control the oscillation rate of the atoms. Thus, the dielectric loss results in the material polarization phase. The dielectric loss of the pure PDMS and PDMS/CB nanocomposite is discussed depending on the ratio of energy lost to the energy supply.

\subsubsection{Effect of volume fraction}

The effect of volume fraction on the pure PDMS and PDMS/CB nanocomposite dielectric loss is shown in Fig. 5. The dielectric loss variability is shown to occur in low, medium, and higher frequency regions. There is a slight change in the loss factor value at the lower frequency range. With the increase in filler content, for medium frequency, constant dielectric loss is observed. However, a substantial increase in dielectric loss is observed at a higher frequency region.

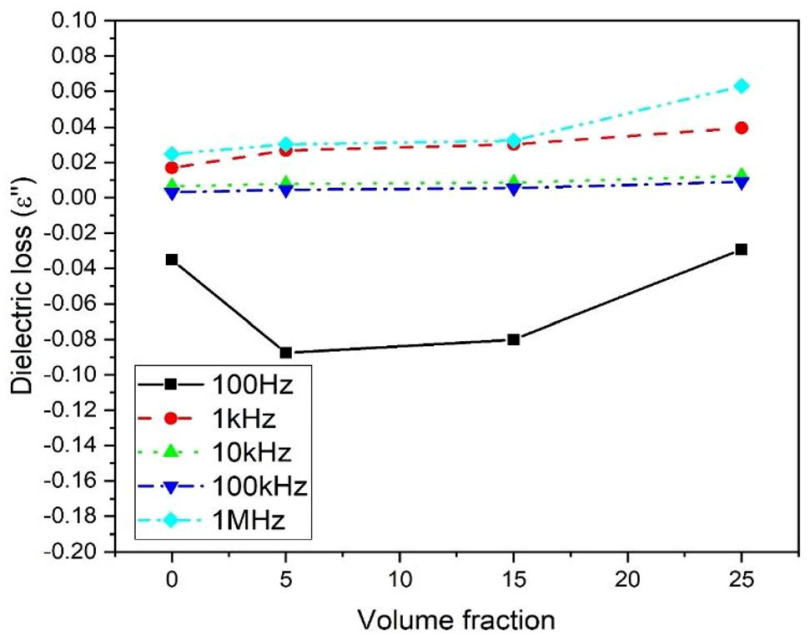

Fig. 5 Variation of dielectric loss as a function of volume fraction of $\mathrm{CB}$

\subsubsection{Effect of frequency}

Dielectric loss is related to the relaxation effect and calculates the exponential decay of polarization over time. The variation in dielectric loss with varying frequencies for pure PDMS and PDMS/CB nanocomposite is shown in Fig. 6. It is noted that there is a steady reduction of dielectric loss with an increase in frequency. The loss value of the nanocomposite is higher than the plain PDMS. The reason could be attributed to the relaxation and an interfacial polarization between the material [43]. There is also a significant increase in dielectric loss due to the higher content of CB-filled PDMS nanocomposite. It may be due to the development of a conductive chain and higher interfacial polarization.

\subsection{Dissipation factor $(\tan (\delta))$}

The dissipation factor or loss of tangent is the ratio of energy lost due to dielectric loss to the energy stored by the dielectric constant during the polarization of the nanocomposite material. The effects of the CB filler and the impact of frequency on the dissipation factor are discussed here.

\subsubsection{Effect of volume fraction}

The volume fraction $\mathrm{CB}^{\prime} \mathrm{s}$ impact on the dissipation factor is shown in Fig. 7. A slight increase in the dissipation factor, with a fractional increase in volume, is noted. The loss factor is minimal at a lower

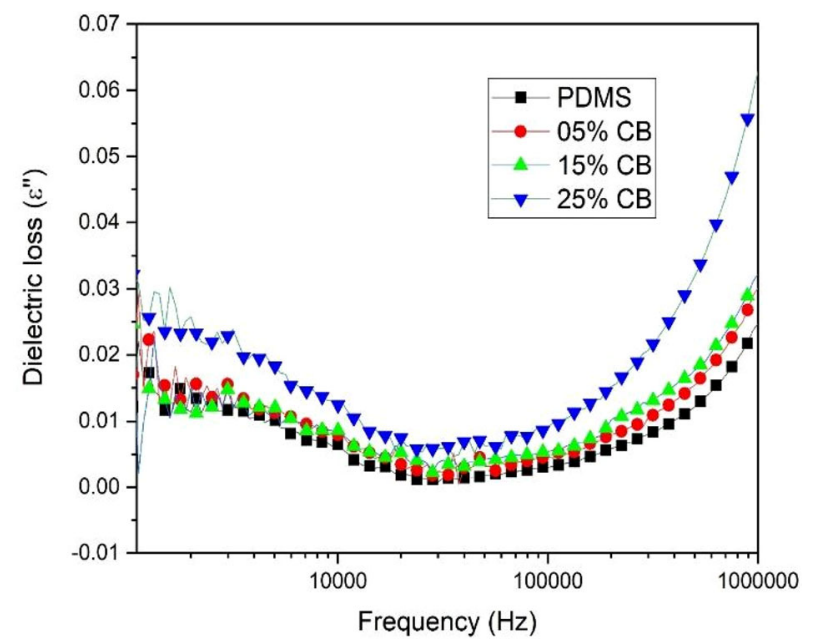

Fig. 6 Dielectric loss versus frequency for the pure PDMS and PDMS/CB nanocomposite 


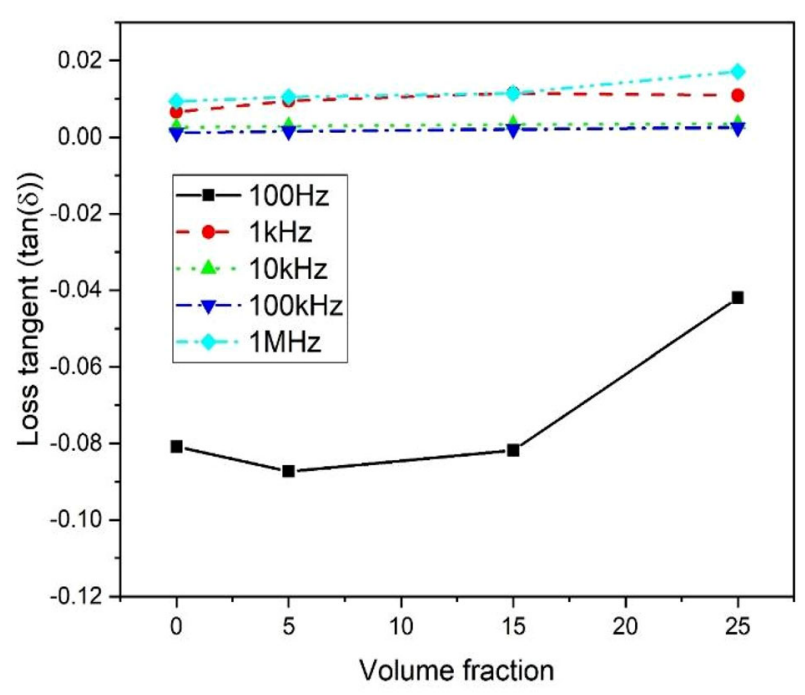

Fig. 7 Loss tangent versus volume fraction of $\mathrm{CB}$ and $\mathrm{PDMS} / \mathrm{CB}$ nanocomposite

frequency, while the loss factor increases significantly at medium frequency. The loss tangent is slightly high at a higher volume concentration of $\mathrm{CB}$; also, the same effect is observed at a higher frequency range. Hence, there are no significant changes in the tangent loss due to filler concentration and higher frequency range. This may be attributed to the non-uniform distribution of conductive particles within the matrix. The degree of interaction between the monomer cross-linker and the conductive filler is significant. As a consequence, there is no systematic pattern of variability for the different volume fractions of fillers.

\subsubsection{Effect of frequency}

The variation of loss tangent of pure PDMS and PDMS/CB nanocomposites with a change in frequency is shown in Fig. 8. It can be seen from the plot that the tangent loss value in the neat PDMS is substantially lower compared to the CB-filled nanocomposite. A small or negligible change of the dissipation value is recorded with an increase in frequency. It is also noted that tangent loss is minimum at the medium frequency range. For frequencies above $100 \mathrm{kHz}$, a steady rise in the loss factor with an increase in frequency is observed. Since the polymer molecules are non-polar in nature and the dielectric relaxation is not due to the dipole orientation, no systematic pattern of variability is observed in all the frequency regions.

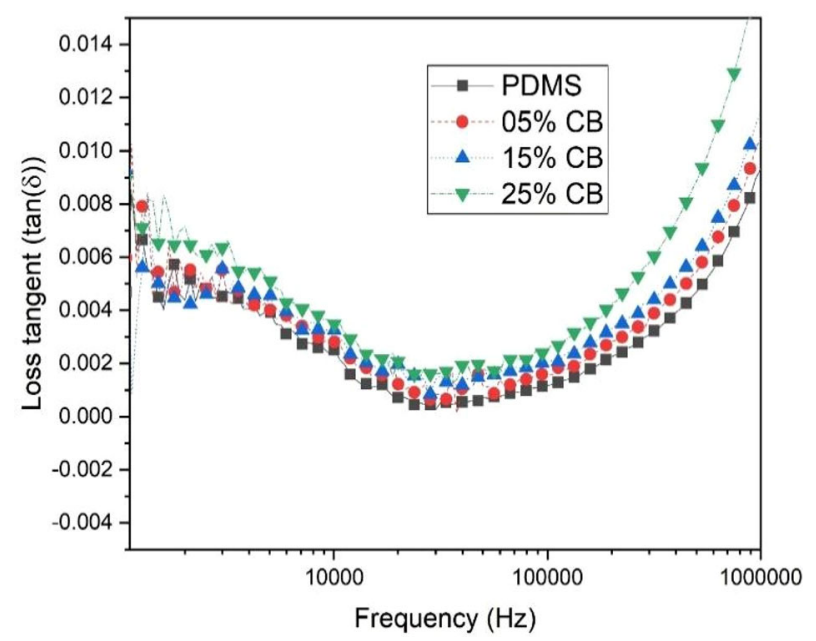

Fig. 8 Loss tangent versus frequency for pure PDMS and PDMS/ CB nanocomposite

\subsection{Prediction of the dielectric permittivity}

Figure 9 shows the effective dielectric permittivity of pure PDMS and PDMS/CB nanocomposites for $1 \mathrm{kHz}$. It is observed that the dielectric permittivity increases with the increase of the volume fraction of carbon black. As seen from the graph, the experimental findings follow the same pattern and fit those of the empirical model. As volume fraction increases, some $\mathrm{CB}$ particles get agglomerated in the nanocomposite. If the particles do not make a continuous chain for conduction, this leads to an underestimation of the composites' effective dielectric constant. It is also noted that there is a significant

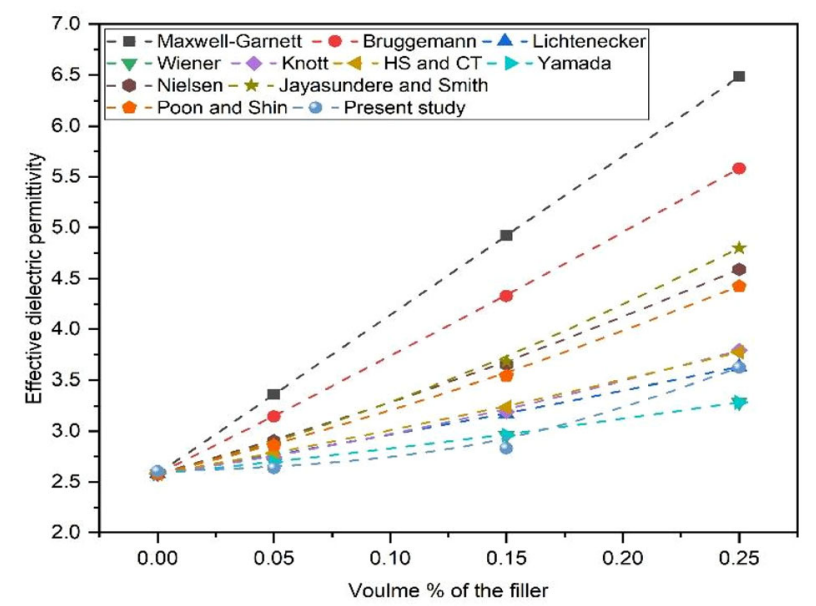

Fig. 9 The effective dielectric permittivity of PDMS/CB nanocomposite with the various volume fractions of $\mathrm{CB}$ 
increase in the effective dielectric constant of the nanocomposite compared to pure PDMS.

Different empirical models were used for illustration and comparison of dielectric permittivity of the nanocomposites. The correlation between theoretical models and experimental results is presented here. In the figure, the theoretical predictions are drawn along with the practical consequence of dielectric permittivity as a function of filler concentration to investigate the agreement between them. According to these models, a nanocomposite's permittivity practically depends on the permittivity of the filler material. Maxwell-Garnett model and Bruggeman model show the big difference with the other models as filler concentration increases. Also, these models do not adequately describe experimental results. Jayasundere-Smith model, Nielsen model, and PoonShin model show much better results than the Maxwell-Garnett model and Bruggeman model because the nanocomposite interactions appear to be significant in their model. Even taking the interaction into account, these models do not prove to be better than the remaining models. Also, these models cannot predict the dielectric permittivity for filler inclusions for the present nanocomposite, as the surface effects become very relevant in the present study. HS-CT model and Knott model exhibit almost linear behaviors as a function of filler concentration. These models overlap with each other and provide good fits to the experimental result.

Similarly, Wiener and Yamada's models also overlap with each other. These models predict almost identical dielectric permittivity of the present study at the lower volume fraction of the filler material. Lichtenecker's model exhibits a very close prediction of dielectric permittivity for higher volume fractions. Out of these models, Wiener, Yamada, and Lichtenecker models are near to the present nanocomposite material.

\subsection{Particle analysis of carbon black}

The procured carbon black material particle size is analyzed using Transmission electron microscopes (TEM). The TEM images of the carbon black filler are shown in Fig. 10. It is observed that the CB particles are almost spherical in shape and a particle has an average size between 100 and $160 \mathrm{~nm}$.

\subsection{Morphology of the nanocomposite}

The dispersion of carbon black in the pure PDMS matrix is observed using a scanning electron microscope (SEM). Figure 11 shows the SEM analysis of the nanocomposite sample of pure PDMS and varied volume percentage of $\mathrm{CB}$ content. It is noticed that the surface of the pure PDMS is in the form of a wavy nature. The distribution of the conducting phase is seen for varied volume percentages $(5 \%, 15 \%$, and $25 \%$ ) of CB content. Further, an increase in CB content shows higher conduction phases and agglomeration of CB particles [22, 44]. Since the uniform dispersion of the $\mathrm{CB}$ is not observed for the nanocomposites with a higher fraction of $\mathrm{CB}$ in the present work, better methods in preparing the composites can lead to further improvement in the dielectric constant [45].

\section{Conclusion}

The present work primarily focuses on evaluating the dielectric behavior of pure PDMS and PDMS/CB nanocomposites. Frequency-dependent dielectric constituents are studied by synthesizing the nanocomposite and experimenting using an impedance analyzer. A significant improvement in dielectric properties was noticed, with an increase in the filler material. The dielectric permittivity, dielectric loss, and dissipation factor of the PDMS/CB nanocomposite show that $\mathrm{CB}$ particle concentration increases the conduction and assists information chain linkage in the nanocomposite material. The effect of volume fraction of the filler and a frequencydependent study show a rise in dielectric permittivity at higher volume percentage and increased sensitivity at low frequency. Also, the addition of different amounts of $\mathrm{CB}$ to the pure PDMS results in varying dielectric behaviors with respect to varying frequencies. Various empirical models were discussed to verify the effective dielectric permittivity of the nanocomposite material. It was observed that the nanocomposites with the increase in filler content obey the model of prediction. Lichtenecker model is very close to the present nanocomposite material at a higher volume fraction. The surface morphology of the nanocomposite reveals a two-phase distribution and agglomeration of particles in the prepared samples. The present investigation can act as a basis for 
Fig. 10 TEM images of carbon black powder
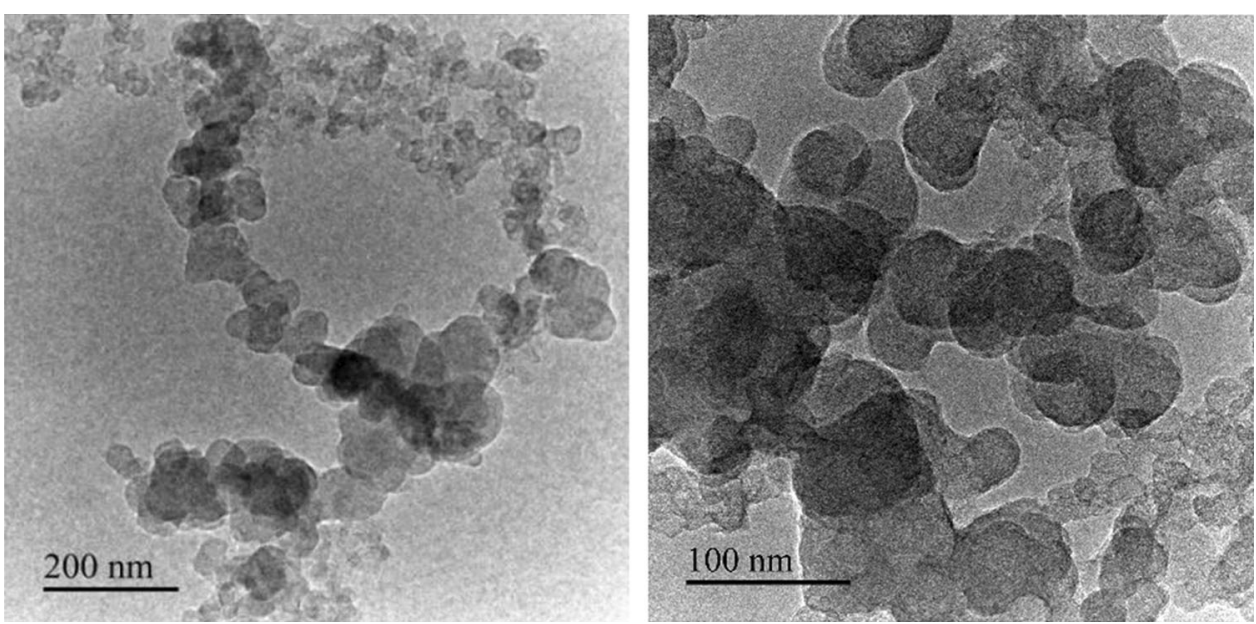

Fig. 11 The SEM analysis of nanocomposite sample with a pure $\mathrm{PDMS}, \mathbf{b} 5 \%$ of $\mathrm{CB}$ content, c $15 \%$ of $\mathrm{CB}$ content, and $\mathbf{d} 25 \%$ of $\mathrm{CB}$ content
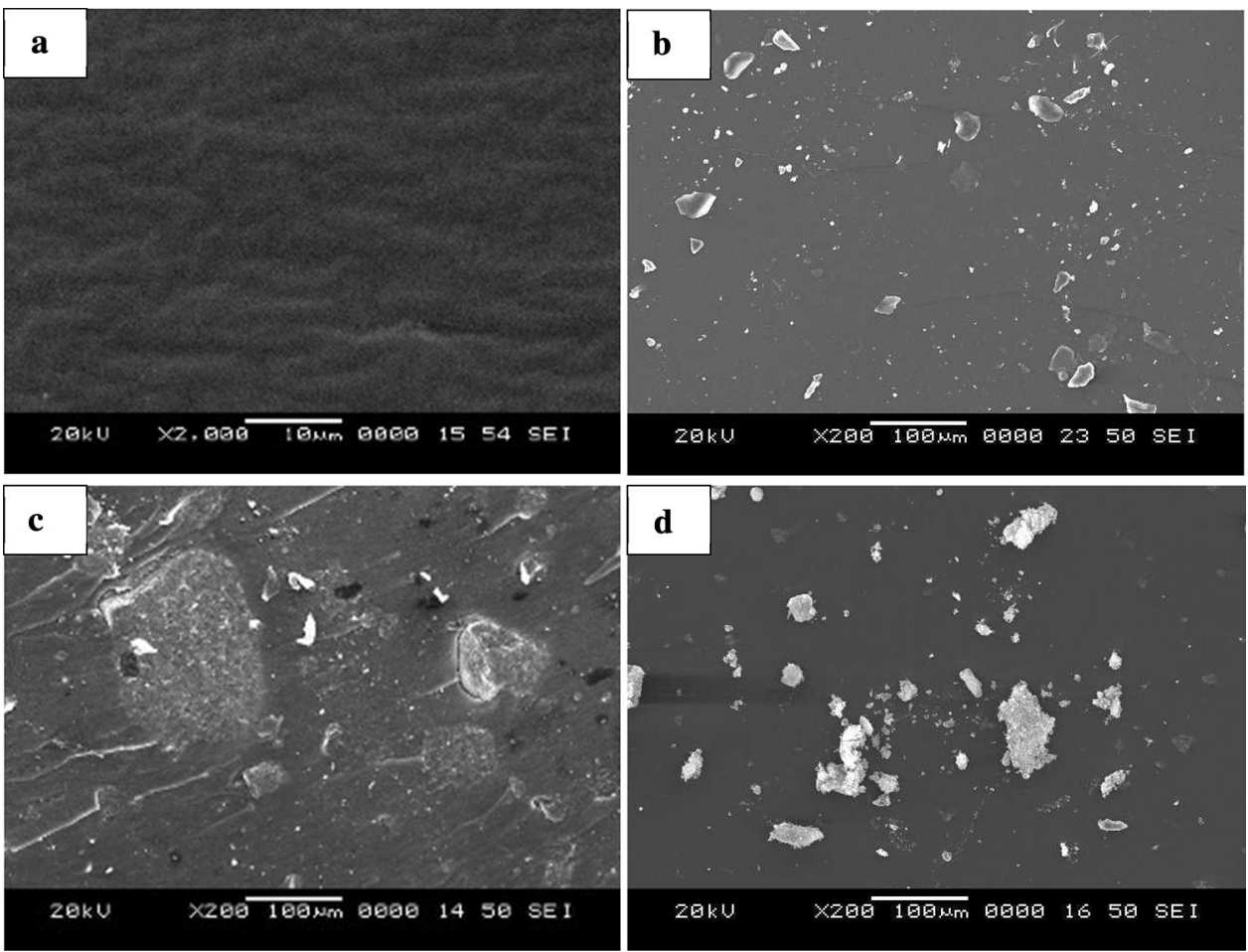

the dielectric analysis of the polymer nanocomposite. Therefore, PDMS/CB nanocomposite may be valid for many applications, such as energy storage and harvesting, electrical and electronic elements, sensor, and actuator.

\section{Acknowledgements}

The authors are grateful to the National Institute of Technology, Rourkela to use dielectric testing, where all experimental measurement is performed. The authors would also like to acknowledge Microsystem Laboratory, Department of Mechanical Engineering, NITK Surathkal for providing the fabrication facility.

\section{Funding}

Open access funding provided by Manipal Academy of Higher Education, Manipal. 
Open Access This article is licensed under a Creative Commons Attribution 4.0 International License, which permits use, sharing, adaptation, distribution and reproduction in any medium or format, as long as you give appropriate credit to the original author(s) and the source, provide a link to the Creative Commons licence, and indicate if changes were made. The images or other third party material in this article are included in the article's Creative Commons licence, unless indicated otherwise in a credit line to the material. If material is not included in the article's Creative Commons licence and your intended use is not permitted by statutory regulation or exceeds the permitted use, you will need to obtain permission directly from the copyright holder. To view a copy of this licence, visit http://creativecommons.org/licen ses/by $/ 4.0 /$.

\section{References}

1. C. Zhang, S. Yin, C. Long, B.W. Dong, D. He, Q. Cheng, Opt. Express 29, 14078 (2021)

2. L. Kolokolova, E. Petrova, H. Kimur, Electromagnetic Waves (InTech, London, 2011)

3. A. Moliton, Basic Electromagnetism and Materials (Springer, Berlin, 2007), pp. 289-315

4. Z. Lou, Q. Wang, Y. Zhang, X. Zhou, R. Li, J. Liu, Y. Li, H. Lv, Compos. Part B Eng. 214, 108744 (2021)

5. M. Hotta, M. Hayashi, M.T. Lanagan, D.K. Agrawal, K. Nagata, ISIJ Int. 51, 1766 (2011)

6. X. Hao, J. Adv. Dielectr. 03, 1330001 (2013)

7. T. Pereira, Z. Guo, S. Nieh, J. Arias, H.T. Hahn, J. Compos. Mater. 43, 549 (2009)

8. Z. Lou, R. Li, P. Wang, Y. Zhang, B. Chen, C. Huang, C. Wang, H. Han, Y. Li, Chem. Eng. J. 391, 123571 (2020)

9. G. Maier, Prog. Polym. Sci. 26, 3 (2001)

10. C. Yang, H. Wei, L. Guan, J. Guo, Y. Wang, X. Yan, X. Zhang, S. Wei, Z. Guo, J. Mater. Chem. A 3, 14929 (2015)

11. X.Y. Zhao, H.J. Liu, Polym. Int. 59, 597 (2010)

12. D. Qiang, Y. Wang, G. Chen, T. Andritsch, IET Nanodielectr. 1, 48 (2018)

13. C. Wu, X. Huang, F. Liu, X. Wu, P. Jiang, in Proceedings of the IEEE International Conference on Solid Dielectrics ICSD (2013), pp. 1091-1094

14. Rashmi, N.M. Renukappa, G. Swaminathan, Siddaramaiah, in Proceedings of the IEEE International Conference on Properties and Applications of Dielectric Materilas (2009), pp. $833-836$
15. M. Rahaman, T.K. Chaki, D. Khastgir, Polym. Eng. Sci. 54, 1632 (2014)

16. H. Yan, Z. Chen, Y. Zheng, C. Newman, J.R. Quinn, F. Dötz, M. Kastler, A. Facchetti, Nature 457, 679 (2009)

17. C. Jean-Mistral, A. Sylvestre, S. Basrour, J.J. Chaillout, Smart Mater. Struct. 19, 075019 (2010)

18. Z.M. Dang, Y. Shen, C.W. Nan, Appl. Phys. Lett. 81, 4814 (2003)

19. E. Tuncer, https://arixv.org/1304.5516 (2013).

20. M.C. Araújo, C.M. Costa, S. Lanceros-Méndez, J. Non. Cryst. Solids 387, 6 (2014)

21. X. Zhang, M.Q. Le, O. Zahhaf, J.F. Capsal, P.J. Cottinet, L. Petit, Mater. Des. 192, 108783 (2020)

22. C. Nawanil, W. Makcharoen, K. Khaosa-Ard, T. Maluangnont, W. Vittayakorn, D. Isarakorn, N. Vittayakorn, Integr. Ferroelectr. 195, 46 (2019)

23. K.K. Sadasivuni, D. Ponnamma, J.-J. Cabibihan, M.A.-A. AlMaadeed, Flexible and Stretchable Electronic Composites (Springer, Cham, 2016), pp. 199-228

24. X.Y. Zhao, H.J. Liu, M.Z. Wang, Recent Advances in Dielectric Materials (IntechOpen, London, 2009), pp. 323-368

25. Z. Ahmad, Polymer dielectric materials. In Dielectric material (Intech Open, 2012).

26. M. Shankar, S. Hiremath, S.M. Kulkarni, Mater. Res. Express 6, 125308 (2019)

27. B. Zazoum, E. David, A.D. Ngo, Trans. Electr. Electron. Mater. 15, 175 (2014)

28. B. Samanta, P. Kumar, D. Nanda, R. Sahu, Results Phys. 14, 102384 (2019)

29. L. Zhang, Z.-Y. Cheng, Progress in Advanced Dielectrics (World Scientific, Singapore, 2020), pp. 369-409

30. K.A. Mathias, H. Shivashankar, B.S.M. Shankar, S.M. Kulkarni, Mater. Today Proc. 33, 5623 (2020)

31. S. Banerjee, K.A. Cook-Chennault, J. Eng. Mater. Technol. 133, 041016 (2011)

32. R. Simpkin, IEEE Trans. Microw. Theory Tech. 58, 545 (2010)

33. M.E. Hossain, S.Y. Liu, S. O’Brien, J. Li, in Technical Proceedings of the 2013 NSTI Nanotechnology Conference and Expo, NSTI-Nanotechnology 2013 1, 554 (2013)

34. A.R. von Hippel, IEEE Trans. Electr. Insul. 23, 801 (1988)

35. E.F. Knott, IEEE Trans. Antennas Propag. 41, 1167 (1993)

36. Z. Hashin, S. Shtrikman, J. Appl. Phys. 33, 3125 (1962)

37. D. Cule, S. Torquato, Phys. Rev. B 58, R11829 (1998)

38. T. Yamada, T. Ueda, T. Kitayama, J. Appl. Phys. 53, 4328 (1982)

39. L.E. Nielsen, J. Phys. D. Appl. Phys. 7, 1549 (1974)

40. N. Jayasundere, B.V. Smith, J. Appl. Phys. 73, 2462 (1993)

41. Y.M. Poon, F.G. Shin, J. Mater. Sci. 39, 1277 (2004) 
42. H. Shivashankar, S.M. Kulkarni, Mater. Sci. Forum 928 MSF, 209 (2018)

43. G. Liu, Y. Chen, M. Gong, X. Liu, Z.-K. Cui, Q. Pei, J. Gu, C. Huang, Q. Zhuang, J. Mater. Chem. C 6, 10829 (2018)

44. S. Gao, H. Zhao, N. Zhang, and J. Bai (2021).
45. R. Han, Y. Liu, J. Shi, G.-X. Chen, Q. Li, E-Polymers 21, 272 (2021)

Publisher's Note Springer Nature remains neutral with regard to jurisdictional claims in published maps and institutional affiliations. 